\title{
EchoGéo
}

16 | 2011

Échos de Turquie

\section{Le pari de la médiatisation de la géographie et de la cartographie}

Entretien avec Frank Tétart

\section{Alexis Sierra}

\section{OpenEdition}

Journals

\section{Édition électronique}

URL : https://journals.openedition.org/echogeo/12402

DOI : $10.4000 /$ echogeo.12402

ISSN : 1963-1197

\section{Éditeur}

Pôle de recherche pour l'organisation et la diffusion de l'information géographique (CNRS UMR 8586)

\section{Référence électronique}

Alexis Sierra, « Le pari de la médiatisation de la géographie et de la cartographie », EchoGéo [En ligne], 16 | 2011, mis en ligne le 04 juillet 2011, consulté le 25 août 2021. URL : http://

journals.openedition.org/echogeo/12402 ; DOI : https://doi.org/10.4000/echogeo.12402

Ce document a été généré automatiquement le 25 août 2021.

EchoGéo est mis à disposition selon les termes de la licence Creative Commons Attribution - Pas d'Utilisation Commerciale - Pas de Modification 4.0 International (CC BY-NC-ND) 


\title{
Le pari de la médiatisation de la géographie et de la cartographie
}

\author{
Entretien avec Frank Tétart
}

\author{
Alexis Sierra
}

1 Frank Tétart est maître de conférences à l'Institut d'Etudes Politiques de Paris, spécialiste des questions européennes et russes ainsi que des nationalismes régionaux. Docteur en géographie et géopolitique de l'Université de Paris 8, diplômé de relations internationales, il s'est fait connaître du grand public en étant le collaborateur de JeanChristophe Victor pour la réalisation de l'émission Le Dessous des Cartes, diffusée sur Arte. Depuis 2009, parallèlement à son travail d'enseignant-chercheur, il est rédacteur en chef délégué de deux magazines: Moyen-Orient et Carto. Le premier, est un trimestriel qui tente d'analyser les enjeux géopolitiques, économiques et sociaux du monde arabo-musulman. Le second présente l'actualité en cartes (voir introduction du dossier). L'interview a été réalisée le 16 décembre 2010.

2 - Alexis Sierra (AS). Frank Tétart, bonjour. Votre parcours professionnel mêle étroitement géographie et journalisme, comment en êtes-vous venu à combiner les deux?

3 - Frank Tétart (FT). Je ne me suis jamais senti totalement géographe ni journaliste. Ma formation avant le doctorat (une maîtrise d'allemand et un magistère en relations internationales de Paris I) n'est pas un cursus classique de géographe, même si j'ai utilisé la géographie assez rapidement sans avoir pour autant suivi un enseignement dès le Deug ${ }^{1}$. C'est donc davantage par une géographie pratique que j'ai commencé ma formation professionnelle et que j'ai appliquée initialement en faisant de la recherche appliquée pour un média télévisuel dans le cadre du Dessous des cartes. Aujourd'hui, après les expériences sur Arte et dans la presse magazine, je peux effectivement être considéré comme journaliste. Or, comme j'utilise à la fois la géographie et la cartographie, avec une visée pédagogique pour décrypter l'actualité, j'ai l'impression de faire non seulement du journalisme en menant des enquêtes, en faisant des interviews, en traitant du factuel mais aussi de la géographie et en proposant des analyses qui sont finalement l'émanation d'une recherche. La différence avec une 
recherche scientifique réellement approfondie tient beaucoup au temps et aux moyens dont je dispose car je ne peux pas passer trois mois sur le terrain. Au final, je continue à me sentir plus un chercheur qui fait du journalisme en appliquant des recettes de géographe et en utilisant la boîte à outils du géographe.

8

- AS. Cette émission était faite pour Arte, c'est-à-dire pour un media qui revendique un niveau d'information et d'analyse assez élevé.

9 - FT. Oui. Cependant, comme le contenu de l'émission était justement assez dense, des cartes « à visée pédagogique » accompagnaient le propos. Elles animaient sur la voix de Jean-Christophe Victor en fonction de ce qui était dit. C'est pour cela qu'il fallait quand même que la carte ne soit pas trop compliquée.

10 - AS. Ce type d'émission, construit autour de cartes, étant nouveau, quelles en étaient les contraintes?

11 - FT. Il y avait des contraintes au niveau technique, par exemple avec les zooms : on n'a pu en faire qu'à partir du moment où les logiciels nous le permettaient parce que le temps de calcul d'un zoom lorsqu'on passe d'une échelle à une autre, du mondial au local par exemple, était très lourd. La machine pouvait parfois calculer pendant 3 heures ou 4 heures! Au final, il était donc plus facile de passer en «cut ", c'est-à-dire d'une image à l'autre en juxtaposé, directement de la petite à la grande échelle, ou vice versa, plutôt que d'être obligé de faire un zoom. Ensuite il fallait re-travailler l'ensemble des typographies parce qu'elles changeaient de taille lors du zoom. Ce sont là des contraintes qui font que les cartes ne devaient pas être trop chargées y compris pour qu'elles restent lisibles.

12 - AS. Quels étaient les éléments représentés? Quels sont les repères choisis sur la carte?

13 - FT. Les reliefs, les frontières, certaines villes, les ressources. Ensuite selon les échelles, on pouvait ajouter un certain nombre d'éléments comme des frontières administratives à l'intérieur des Etats ainsi que les noms de pays voisins. Quand on faisait une carte de la Turquie par exemple, on inscrivait autour les pays voisins pour qu'on se repère dans l'espace. Cela permettait de souligner le fait que la Turquie est proche du Moyen-Orient parce qu'elle a une frontière avec l'Iran / l'Irak tout en étant en Europe, avec de l'autre coté une frontière terrestre commune avec la Roumanie ou la Grèce. Aussi, rien qu'en voyant la carte, le spectateur pouvait constater que la Turquie est au croisement de l'Orient et de l'Europe. C'est ce genre d'élément simple qui permettait de situer géographiquement un territoire dans un espace régional ou sous régional.

14 - AS. Une des contraintes majeure dans les media audiovisuels, c'est le temps. Avec des conséquences sur l'analyse développée?

15 - FT. Le Dessous des cartes était en effet une émission courte. C'était plutôt un choix ... Au départ, quand j'ai commencé, elle durait moins de 6 minutes. Ensuite, elle est passée à 8 
minutes, puis à 11 soit le double de temps, ce qui permettait d'en dire un peu plus, de dé - densifier et puis d'avoir un raisonnement plus abouti. D'un autre coté, il ne fallait pas que ce soit trop long: en 10 minutes, on peut faire le point sur une question. En revanche, vu la densité de l'information contenue dans l'émission, je ne suis pas certain que plus long aurait été positif. Un auditeur, un téléspectateur, décroche au bout d'un moment. Même s'il y a une animation cartographique derrière, sa concentration faiblit car ce sont malgré tout des sujets complexes. Quand on traite du Moyen-Orient, quand on traite de situations géopolitiques comme au Soudan, que les gens connaissent mal, ou même de la Colombie, avec des groupes particuliers comme les Farc, en 10 minutes, même si la carte éclaire, cela reste complexe. Je ne suis pas sûr qu'au-delà de 15 minutes d'antenne, les gens continueraient à suivre. Alors qu'avec une dizaine de minutes, cela permet d'avoir une petite introduction dans laquelle l'animateur présente le thème et en donne les enjeux. Le déroulé des cartes animées dure ensuite 8 minutes environ. Il reste alors $1 \mathrm{mn} 30$ de conclusion durant lesquelles Jean-Christophe Victor réapparaît à l'antenne donnant un certain nombre de pistes ainsi qu'une ou deux références bibliographiques pour donner des pistes d'approfondissement.

- AS. Comment était perçue l'émission par le reste de la Châ̂ne ? Parce que s'il a fallu accroitre de quelques minutes le temps d'antenne, je suppose qu'il a fallu argumenter.

17 - FT. Petit à petit, la Chaîne, suite aux demandes, a accepté d'allonger le format. Au départ c'était donc 5 à 6 minutes; on est passé dans un premier temps à 7/8 minutes et dans un seconde temps à 10/11 minutes. Cela s'est fait par petites étapes, en justifiant effectivement que cet accroissement de la durée permettait d'avoir une petite souplesse sur la démonstration et un débit moins rapide. Beaucoup de téléspectateurs écrivaient en effet pour dire que ça allait souvent trop vite. C'était donc un argument qui nous permettait de proposer un format plus adapté à l'analyse de ces situations complexes.

18 - AS. Pour finir sur Le Dessous des cartes, comment s'est décidé le passage au DVD et à l'atlas qui sont d'autres formes de communication en somme?

19 - FT. Le DVD, c'était avant tout une démarche commerciale de Arte. Comme l'émission avait du succès, la Chaîne avait envie de faire des produits dérivés et donc le DVD (après les cassettes VHS). Rapidement, on a proposé de favoriser une approche régionale : un premier DVD regroupait, sur les deux dernières années de diffusion, les émissions sur l'Europe, sur l'Asie ou sur l'Afrique. Ce n'était pas une approche exhaustive, mais elle permettait d'avoir une cohérence géographique et de donner un certain nombre de clés de lecture sur une zone. Pour nous, au final, faire un DVD avait de l'intérêt car cela permettait de se rendre compte du contenu des émissions. Par exemple, on pouvait s'apercevoir que sur telle ou telle région, certains espaces ou Etat n'avaient pas été traités.

20 La production d'un atlas relevait d'un autre contexte. Beaucoup de spectateurs écrivaient et demandaient: "Est-ce que Le Dessous des cartes a des publications? Le Dessous des cartes va-t-il faire un atlas ?". Jean-Christophe Victor a commencé à y réfléchir puis l'équipe de production de Arte s'y est intéressée. Cependant, ce fut assez fastidieux de trouver un éditeur. Cela peut apparaitre paradoxal mais en dépit du fait que les émissions et les cartes existaient, l'éditeur s'est rendu compte qu'il fallait...refaire les cartes !

21 Si on voulait quelque chose de qualité, il fallait refaire les cartes puisque les cartes, c'étaient des cartes en mode vidéo qui n'étaient pas récupérables pour un ouvrage 
papier. Du coup, ce fut une contrainte avec évidemment un coût. C'est ainsi qu'on a eu plusieurs propositions d'éditeurs qui ont finalement échoué jusqu'à ce que Tallandier dise : "mais ça va marcher, c'est un beau projet, les gens aiment bien les atlas et les cartes; en plus votre approche, c'est quand même de vulgariser la géographie, d'apporter les réponses au plus grand nombre sur les grandes questions internationales et ça, je pense que ça va marcher". Et effectivement ça a marché !

- AS. Avec en outre une véritable utilisation pédagogique puisque, que ce soit les DVD ou les atlas, on les retrouve dans les lycées, les collèges, les bibliothèques.

23 - FT. Je pense qu'un des atouts des DVD, c'est que le chapitrage permet une approche pédagogique aisée en laissant le choix des entrées. Les professeurs ont beaucoup apprécié parce qu'ils pouvaient faire eux même une introduction sur telle ou telle région puis montrer $2 \mathrm{mn} 30 \mathrm{du}$ Dessous des cartes, puis faire une pause, expliquer ce qu'ils avaient vu aux élèves et reprendre le cours. Ils pouvaient ne montrer qu'un ou deux aspects en fonction des sujets, en fonction des programmes de l'Education Nationale.

24 - AS. Aviez-vous eu des retours sur l'utilisation de la géographie dans les media ? Cette émission a-t-elle favorisé le goût pour la géographie ? Est ce que tout d'un coup, c'est une discipline qui pouvait apparaître comme plus attractive?

25 - FT. Je crois que c'est assez vrai car dès qu'il y a eu des papiers écrits par différents journalistes pour présenter l'émission ou pour présenter les DVD, c'était à chaque fois un des points mis en avant : grâce à l'émission Le Dessous des cartes, les Français vont aimer la géographie ou vont l'aimer parce que, justement, il y a cette dimension pédagogique avec l'utilisation de cartes qui non seulement étaient assez belles et colorées mais pouvaient être assez simples à lire.

Du coup, et c'est le point le plus intéressant, les spectateurs se rendaient compte qu'il y avait énormément de phénomènes qui étaient spatialisables et que, par conséquent, la carte de géographie avait une véritable dimension politique pour comprendre un certain nombre de situations.

27 Pour comprendre ce relatif succès, il faut aussi rappeler le contexte de création de l'émission en 1990/91 c'est-à-dire au moment de la guerre du Golfe. Il y avait eu la fin de la guerre froide, cette guerre du Golfe qui arrive là-dessus, la fin de l'Union Soviétique quelques mois plus tard, la réunification allemande... Il y avait une recomposition des frontières à l'échelle européenne. On entendait parler de nouveaux Etats, les pays baltes, la Moldavie, le Turkménistan, le Kazakhstan, tous les pays en -stan d'Asie centrale et les gens ne savaient pas trop ce qu'ils étaient. Ils avaient oublié leurs cours d'histoire et certains Etats naissaient sur la carte de l'Europe pour la première fois, notamment tous les pays issus de l'Union soviétique et ensuite issus de la décomposition yougoslave puis de celle de la Tchécoslovaquie.

Dans ce contexte, Le Dessous des cartes apportait un certain nombre de clés de lecture ou tout simplement montrait où se situait tel ou tel Etat méconnu, dans quelle dynamique il se trouvait et quelles évolutions cela impliquait.

29 - AS. Alors justement, on pourrait être surpris qu'il n'y ait pas davantage d'émissions à la télévision autour des cartes ou de l'analyse géographique ou géopolitique. En avezvous parlé dans l'émission? Certes, il y a toujours une petite carte de localisation dans les actualités ou dans certaines émissions mais de là à avoir une émission consacrée à la géographie du monde, à la géopolitique, à l'utilisation des cartes, il y a un pas qui ne 
semble pas franchi. Or il y a toute cette dimension visuelle que vous avez mis en valeur, exploité, montré et qui est en phase avec l'audiovisuel ainsi que toute cette dimension pédagogique, cette utilité pour comprendre l'actualité dont on nous bombarde. Est-ce que vous avez eu une démarche un peu réflexive en disant : « voilà ce qu'on a fait, nous, avec Le Dessous des cartes, est ce que ça existe par ailleurs, est ce que il n'y aurait pas d'autres choses à faire»?

- FT. On avait regardé un peu ce qui existait ailleurs et je crois que dans le Monde c'était la seule.

- AS. Dans le Monde?

- FT. Oui, dans le Monde! La seule émission dont le seul et unique support soit des cartes, c'est celle-là. Après, effectivement, on voit la multiplication des cartes un petit peu partout dans les media, y compris dans les media papier comme en témoigne le succès de tous les atlas que ce soit celui du Monde diplomatique ou du Monde quotidien qui sort désormais un atlas thématique deux fois par an. On voit bien l'essor que la cartographie a pris. Maintenant, en termes de media télévisuel, j'avais fait une recherche pour mon DEA en 2000 à l'Institut français de Géopolitique avec Béatrice Giblin. J'avais analysé l'originalité du Dessous des cartes justement, de l'approche géographique à la télé, dans les media. Je n'avais alors rien trouvé. Le seul qui travaillait pour une chaîne de télévision, c'était un géographe canadien, Rodolphe de Koninck qui faisait en fait un cours de géographie pour ses étudiants en étant filmé. Ensuite, le cours était retransmis sur une chaîne du savoir et de la connaissance qui avait plus ou moins une vocation d'enseignement par correspondance sur les télévisions du Québec. C'était donc un cours de géographie, dans lequel il montrait effectivement un certain nombre de cartes qu'il utilisait et dans lequel il avait généralement une carte derrière lui. Mais c'étaient les seules choses que j'avais pu repérer jusqu'en 2000.

revanche, c'est vrai que depuis une bonne dizaine d'années, voire 15 ans, on constate la multiplication des cartes dans les media. Le Monde montre quasiment tous les jours une ou deux cartes; de même pour le Figaro. Il y a beaucoup plus de cartes qu'avant, qui ne sont pas seulement des cartes de localisation. Quand, dans Le Monde, on a une carte du Soudan qui montre les enjeux de la partition avec l'éventuelle frontière, les zones de pétrole, on est déjà dans la carte thématique. Et non plus dans la carte de localisation du journal télévisé pour repérer le lieu d'un fait divers. Désormais, il y a de vraies cartes thématiques, ce qui de mon point de vue est un grand changement. J'y vois la volonté d'expliquer un certain nombre d'enjeux sur des cartes parce que ces enjeux sont spatialisables et que ce sont des enjeux territoriaux et donc géopolitiques.

Il y a aujourd'hui une émission qui a pris Le Dessous des cartes comme modèle, de l'avis même de l'animateur. C'est une émission de BFM TV la chaîne d'information du Câble donc. Harold Himan, un franco-américain, explique tel ou tel fait de l'actualité avec une grande carte derrière lui et quelques animations un peu comme un maître d'école. L'émission est quotidienne et dure $2 \mathrm{mn}$ environ avec une carte dans le dos.

- AS. La faiblesse du nombre de ce genre d'émission s'explique-t-elle par son coût ?

- FT. Faire des cartes n'est pas simple. Il faut être un peu géographe ou beaucoup cartographe. Cela ne consiste pas seulement à recopier un atlas. Si on veut montrer un certain nombre d'enjeux, il faut croiser des informations, il faut faire un peu de recherche de fond. C'est donc assez cher. Il faut acheter des fonds de carte et des droits 
de diffusion : à la télévision, tout doit se payer. Il faut donc non seulement acheter un fond de carte mais il faut aussi payer le droit de l'utiliser sur une chaîne de télé. Ensuite, il faut payer un infographiste pour faire les éléments cartographiques. La carte animée n'est pas évidente à faire comme je l'indiquais précédemment. C'est court mais cela représente beaucoup de travail en amont.

Nonobstant, ce n'est pas la seule raison qui bloque la diffusion de ce type d'émissions. Je ne connais pas les autres raisons mais j'imagine que TF1 est peut être moins intéressée par les cartes que par un reality show. Ce sont aussi des stratégies commerciales qui entrent en jeu.

- AS. Vous avez arrêté de travailler pour le Dessous des cartes en 2008 et vous êtes passé à un autre type de media, en devenant rédacteur de deux revues. Comment s'est passé le passage de la télévision au papier?

39 - FT. La revue Moyen Orient a été lancée en 2009 et Carto en 2010. J'étais déjà dans le bain de la cartographie et dans tout ce qui était géographie « appliquée » et médiatisée. Même si j'enseigne, c'est vrai que j'étais déjà dans ce domaine là. J'avais déjà approché un autre support en passant à l'écrit avec les atlas. Donc du coup quand je suis passé à la presse, sur Moyen-Orient puis sur Carto, j'avais déjà une expérience pour faire quelque chose en relation avec ce support.

40 C'est Alexis Bautzman que je connaissais un peu qui m'a proposé de travailler chez Areion. Il voulait absolument une revue sur le Moyen-Orient pour commencer. Au bout de quelques mois, il m'a présenté un autre projet sur lequel il réfléchissait depuis un moment : une revue sur l'actualité vue par les cartes. Il m'a demandé si j'étais intéressé pour mener ce projet : «Est ce que ça t'intéresse ? » Evidemment, je n'ai pas dit non!

41 - AS. Est-ce plutôt une revue de vulgarisation?

42 - FT. C'est plutôt un magazine. Tous les deux mois, nous reprenons un certain nombre de sujets d'actualité avec des cartes éclairant les enjeux. On retrouve un peu l'esprit de ce que j'avais fait dans Le Dessous des cartes, avec un accompagnement de textes qui sont assez courts, qui donnent un certain nombre d'informations soulignant les enjeux de tel ou tel pays, ou de telle ou telle crise, ou de tel ou tel thème. C'est un travail d'équipe notamment avec une cartographe, Laura Margueritte, avec qui je conçois des cartes qui spatialisent ces informations. Il y a un vrai échange, un travail à la fois de réflexion par rapport à une analyse territoriale et esthétique autour de la carte.

43 C'est une autre façon d'appréhender l'actualité : quand on regarde les actualités, soit à la télévision, soit dans la presse, ce sont plutôt des exposés factuels mais les analyses de fond, qui reviennent un petit peu sur l'histoire, qui prolongent sur les enjeux économiques, qui prolongent sur les enjeux politiques sont plus rares. L'idée pour nous est également d'avoir une approche prospective qui essaie de tirer la réflexion vers le territoire, vers les tensions sur des espaces géographiques.

Nous avons également plus de temps puisque nous fabriquons un bimensuel. Nous pouvons avoir un petit peu de recul pour réagir à l'actualité et voir comment on peut la cartographier.

45 - AS. A combien d'exemplaires, est édité le mensuel ?

46 - FT. 15000 numéros sont tirés. Je n'ai pas les chiffres exacts des ventes, mais cela tourne autour 6000 exemplaires pour le premier numéro. Comme quoi la géographie intéresse encore un public. C'est plutôt encourageant. 
47 - AS. Je vais renverser la question. Analyser, décrypter l'actualité, vous pouvez le faire aussi bien comme chercheur que comme enseignant. Quel est l'apport de travailler pour un mensuel?

48 - FT. Dans ce travail, il faut essayer d'avoir une approche globale que je dois ensuite essayer d'ingérer, de comprendre et que je dois enfin transmettre à un public large en hiérarchisant les informations qui sont les plus importantes. Finalement, je dois essayer de faire l'entonnoir.... Cela m'apporte beaucoup en termes pédagogiques car je dois formaliser, hiérarchiser l'information, choisir l'information qui, moi, me paraît la plus pertinente pour comprendre une actualité. Je prends un exemple récent : la crise entre les deux Corées. [l'interview a été réalisée le 16 décembre 2010, peu de temps après le bombardement par la Corée du Nord d'un îlot frontalier avec la Corée du Sud et la surenchère verbale qui s'en est suivie]. Les media ont beaucoup parlé de la Corée mais il y a deux ou trois faits à connaître pour essayer de comprendre la crise: 1. C'est le contexte historique, c'est-à-dire pourquoi la péninsule est divisée et depuis quand. 2. Pourquoi aujourd'hui il y a ces gesticulations autour de la Corée du Nord? Parce qu'ils sont très isolés et que face à eux, il y a la Corée du Sud qui est soutenue par les Etats-Unis, donc première puissance mondiale. Au final, tout cela renforce une perception d'encerclement ( $3^{\mathrm{e}}$ facteur). La Corée du Nord va donc utiliser l'alibi nucléaire ou envoyer une bombe sur un îlot où il $\mathrm{y}$ a peu de personnes. Cela pour dire aux Américains, qu'ils existent et qu'ils comptent sur la scène internationale. Si les Américains n'étaient pas en Corée du Sud, il y aurait peut-être moins de gesticulations de la Corée du Nord. Nous avons donc 3 ou 4 données d'ordre géographique qui éclairent la situation. Or, le plus souvent on va entendre les faits : voilà, il s'est passé telle agression, certes en localisant sur la frontière, à tel lieu mais sans aborder la profondeur historique ni tous les enjeux qui en découlent. Je crois que la carte permet de simplifier un certain nombre de choses en les spatialisant. Lorsqu'on voit le positionnement des bases américaines, on comprend assez bien que, sans vouloir défendre la Corée du Nord, il y a une perception d'encerclement de la Corée du Nord. Elle se sent assiégée et elle va instrumentaliser cette position d'isolement en s'adressant aux Etats-Unis de façon conflictuelle, d'autant qu'il s'agit de la première puissance au monde.

49 - AS. Pour la revue Moyen-Orient, faîtes-vous appel à des géographes ?

50 - FT. Oui. La difficulté est que pour certaines zones, il y a peu de spécialistes en géographie: sur l'Iran, il y a par exemple Bernard Hourcade, mais sur l'Algérie ou l'Egypte, il y a peu de chercheurs reconnus. Nous faisons alors appel à des politistes. Et quand il existe des géographes sur ces terrains, ils abordent peu les questions politiques qui sont des sujets sensibles dans ces pays. Or, notre objectif est d'aborder prioritairement ces questions.

51 - AS. Vous enseignez comme maître de conférence à Science Po Paris ; c'est une école qui forme notamment des journalistes. Quelle est la situation de la géographie dans le cursus de Science Po?

52 - FT. Sciences Po a un atelier de cartographie qui est à la disposition des étudiants. Je ne sais pas s'il y en a beaucoup qui s'y rendent même si pour ma part, j'oblige les étudiants à présenter des exposés avec des cartes pour les habituer à spatialiser les événements. Cependant, depuis la rentrée 2010-2011, l'enseignement de géographie de première année a été supprimé après être devenu optionnel alors qu'au départ, il était obligatoire. 
53 - AS. C'est contradictoire avec la dynamique que nous évoquions tout à l'heure, celle qui voit la cartographie et l'analyse géographique se développer dans les media et de l'autre côté, la réduction de l'enseignement de la géographie dans une filière qui a pour vocation d'étudier l'actualité. Comment se fait-il que la géographie soit passée à la trappe?

54 - FT. Ce qui nous a été présenté, c'est la volonté de procéder à un recentrage sur les fondamentaux de Sciences Po: en première année, les sciences politiques, l'histoire et l'économie. En deuxième année, les étudiants s'ouvrent aux relations internationales et par conséquent, ils vont faire de la géographie à travers les relations internationales.

55 Du coup, j'ai proposé un enseignement de substitution, puisque les étudiants peuvent prendre un certain nombre d'options durant les premières années, sur la géopolitique de l'Europe. D'autres collègues font également aussi des approches en géographie sur des espaces réduits (les villes, le Moyen-Orient, l'Afrique etc.). De manière optionnelle, il y aura donc encore un certain nombre d'étudiants qui pourront faire de la géographie mais plus de caractère obligatoire ni d'option obligatoire comme l'année dernière où il $y$ avait en gros $50 \%$ qui faisait de la géographie et $50 \%$ qui faisait d'autres options.

56 - AS. Donc la suppression ne s'explique pas par le fait qu'il y avait un désintérêt?

57 - FT. Non. Je pense que c'est une volonté de recentrer et de reformatage du programme de première année parce que certains étudiants avaient des lacunes en sciences politiques ou en sociologie politique notamment et qu'ils ont voulu renforcer cette formation. C'est quand même un institut dédié aux sciences politiques. Toutefois, je pense que c'est quand même un peu dommage qu'une institution comme Sciences Po, qui va former ensuite des politiques et des journalistes, n'ait pas d'enseignement de géographie obligatoire en première année. Après, en seconde année, ils vont aborder les questions géographiques mais à travers le prisme des relations internationales et de la mondialisation et non plus avec une approche spécifiquement géographique comme on le faisait jusqu'à récemment.

58 - AS. Puisse votre action comme rédacteur de Carto faire réfléchir au rôle central de la géographie dans les sciences politiques! En attendant, merci de nous avoir montré comment peut s'opérer cette croisée des chemins entre géographie et journalisme.

\section{NOTES}

1. Anciennes premières années de licence. 


\section{AUTEUR}

\section{ALEXIS SIERRA}

Alexis Sierra est maître de conférences en géographie à l'université Cergy-Pontoise-IUFM. alexisierra2001@yahoo.fr 